\title{
Statistic analysis of swarm activities around the Boso Peninsula, Japan: Slow slip events beneath Tokyo Bay?
}

\author{
Tsubasa Okutani ${ }^{1}$ and Satoshi Ide $^{2}$ \\ ${ }^{1}$ Earth and Planetary Physics, Univ. of Tokyo \\ ${ }^{2}$ Earth and Planetary Science, Univ. of Tokyo \\ (Received March 24, 2010; Revised January 5, 2011; Accepted February 21, 2011; Online published June 21, 2011)
}

\begin{abstract}
Seismicity re ects underground stress states, satisfying scaling laws such as Gutenberg-Richter law and OmoriUtsu law. Standard seismicity models based on these scaling laws, such as the Epidemic Type Aftershock Sequence (ETAS) model, are useful to identify swarm anomalies in seismicity catalogs. Llenos et al. (2009) applied the ETAS model to swarms triggered by slow slip events (SSEs) and found that stressing rate controls the background seismicity $\mu$ suggesting that swarms can be utilized to monitor stress change due to various aseismic processes. Following their work, we analyze the 2002 and 2007 Boso swarms triggered by the Boso SSEs (Ozawa et al., 2007) and a swarm beneath Tokyo Bay, in June 2005. A single ETAS model cannot explain the high seismicity during a swarm. Although a combination of three ETAS models for pre-swarm, swarm, and post-swarm periods better explains the data, a simpler model with an ETAS model and a boxcar function is even better. Similarity of the seismicity model, together with the locations and focal mechanisms, suggests that three swarms share a common source of stress, and the possibility of undetected SSE beneath Tokyo Bay.
\end{abstract}

Key words: Swarm, seismicity, slow slip event, ETAS model, Boso Peninsula.

\section{Introduction}

An earthquake swarm is de ned as a period of high seismicity without a distinguished mainshock. Despite its prevalence in earthquake catalogs, the background physics still remains unclear (e.g., Vidale and Shearer, 2006). Some swarms are induced by static stress change due to volcanic eruptions, such as the 2000 Izu Islands, Japan swarm (e.g., Toda et al., 2002) induced by the eruption of Miyake Island volcano. The diffusive migration of uid is also a plausible mechanism, as suggested for the 1965-67 Matsushiro (Ohtake, 1974) and the 1998 Hida (Aoyama et al., 2002) swarms, Japan. Slow slip events, SSE, are another kind of the swarm driving mechanism. Recently, SSE induced swarms have been discovered in various geological environments, such as near the San Andreas Fault, California (Lohman and McGuire, 2007), beneath Kilauea volcano (Montgomery-Brown et al., 2008), Hawaii (Segall et al., 2006), and in subduction zones in Japan (Ozawa et al., 2007; NIED, 2007) and New Zealand (Reyners and Bannister, 2007).

To investigate swarms as anomalies in seismicity cata$\log$, it is important to de ne the standard seismicity. The Epidemic Type Aftershock Sequence (ETAS) model (e.g., Ogata, 1988, 1999) is one of standard seismicity models and explains the characteristics of seismicity based on a simple assumption that an earthquake sequence is a point process with time-dependent seismicity rate. Each earth-

Copyright (C) The Society of Geomagnetism and Earth, Planetary and Space Sciences (SGEPSS); The Seismological Society of Japan; The Volcanological Society of Japan; The Geodetic Society of Japan; The Japanese Society for Planetary Sciences; TERRAPUB.

doi:10.5047/eps.2011.02.010 quake produces aftershocks following the Omori law and the Gutenberg-Richter law. Llenos et al. (2009) applied the ETAS model to SSE-triggered swarms in three regions and found that the background seismicity rate increases by orders during SSE without signi cantly changing other characteristics. This property of SSE-triggered swarm may be useful to identify smaller SSE undetectable using current geodetic observation systems.

One of the study areas in Llenos et al. (2009) is beneath the Boso Peninsula, Japan (Fig. 1), where the Philippine Sea plate is subducting northward generating megathrust earthquakes of magnitude about 8 , such as the 1923 Kanto earthquake. SSEs occurred in this area at about 5 year recurrence interval. Ozawa et al. (2007) determined slip models using GPS records of recent three events occurred in 1996, 2002, and 2007. Although the spatio-temporal slip evolution is not exactly identical, the sizes of event are comparable and between $M_{\mathrm{w}} 6.4$ and 6.6. In the northwest of these $M \sim 6.5$ SSEs and swarms, there were minor swarms beneath Tokyo Bay, right beneath Tokyo Metropolis. Here we investigate the stochastic property of the Boso Peninsula and Tokyo Bay swarms based on ETAS analysis following Llenos et al. (2009). The facts that swarms in two areas are explained by a simple swarm model and that they occur near the plate interface with low-angle thrust mechanism imply the possibility of undetectable SSE beneath Tokyo Bay.

\section{Methods}

To evaluate the genuine effect of external events like SSE on seismicity, we need to remove the seismicity due to mainshock-aftershock sequences from the seismicity catalog. We adopt Epidemic Type Aftershock Sequence (ETAS) 


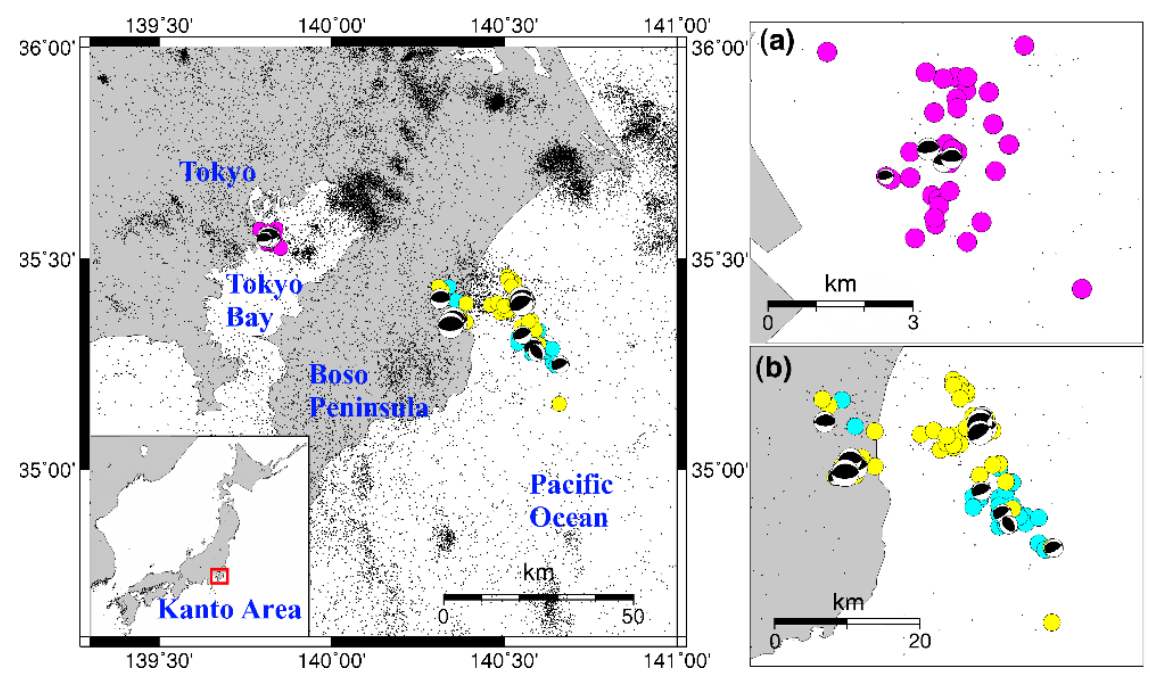

Fig. 1. The earthquake distribution beneath the Boso Peninsula. The purple circles represent the Tokyo Bay swarm in 2005 . The cyan and yellow circles represent the Boso swarms in 2002 and 2007 respectively. Focal mechanisms determined by JMA for these swarm events are shown by beach balls. Black dots represent earthquakes, which occurred at depths from $0 \mathrm{~km}$ to $50 \mathrm{~km}$ between 2000 and 2008 . Zoom up figures are shown in the right panels. (a) and (b) show the Tokyo Bay and Boso swarms respectively.

model (e.g., Ogata, 1988, 1999) as a standard seismicity model and characterize swarms as anomalies from the standard. ETAS model is a point process model, including the Omori-Utsu law (Utsu, 1961; Utsu et al., 1995) and branching process in which every earthquake has some ability to generate its own aftershocks. Therefore, the seismicity rate at time $t$ is given by summing the effects of all prior earthquakes and background seismicity rate $\mu$ as,

$$
\lambda(t)=\mu+\sum_{t_{i}<t} K \exp \left(\alpha\left(M_{i}-M_{0}\right)\right) /\left(t-t_{i}+c\right)^{p},
$$

where $c$ and $p$ are parameters in the Omori-Utsu law, $K$ and $\alpha$ control the aftershock productivity by a mainshock and its magnitude sensitivity, respectively. These five parameters, $\mu, c, p, K$, and $\alpha$, are determined by Maximum Likelihood Estimation (MLE) with an earthquake catalog consisting of hypocentral times $t_{i}$ and magnitudes $M_{i} . M_{0}$ is the reference magnitude, which we equalize to the catalog threshold magnitude $M_{\mathrm{c}}$.

MLE is a practical method for fitting a stochastic model to a data set. In ETAS model, the log likelihood can be expressed as follows:

$$
\log L=\sum_{t_{i} \leq T} \log \lambda\left(t_{i}\right)-\int_{0}^{T} \lambda(t) d t
$$

where the integration is calculated from the beginning, 0 , to the end, $T$, of the data set. We find out a parameter set that maximizes the log likelihood by the Broyden-FletcherGoldfarb-Shanno method (e.g., Fletcher, 1980).

To analyze a seismicity catalog including a period with anomalous activity, i.e. swarms, it is necessary to consider how to characterize the period. If a period is divided into two sub periods and two models are used to explain them, the higher degree of freedom allows apparently better explanation of data. This is a change point problem and the goodness of fit should be measured by another criterion, Akaike Information Criterion (AIC). For a single model with $k$ free parameters, AIC is defined as

$$
\mathrm{AIC}=-2 \log \hat{L}+2 k,
$$

where $\hat{L}$ is the maximum likelihood. If there are several models with different numbers of parameters, the model that gives the smallest AIC is the best model. The approach that selects the smallest AIC model is called Minimum AIC Estimation (MAICE).

When we consider two sub periods, the goodness of models with a time interval $[S, T]$ and two intervals $\left[S, T_{0}\right]$ and $\left[T_{0}, T\right]$ are respectively measured using AIC values,

$$
\begin{aligned}
& \mathrm{AIC}_{0}=-2 \log \hat{L}_{0}(S, T)+k_{0}, \\
& \mathrm{AIC}_{1}=-2 \log \hat{L}_{1}\left(S, T_{0}\right)+k_{1}, \\
& \mathrm{AIC}_{2}=-2 \log \hat{L}_{2}\left(T_{0}, T\right)+k_{2},
\end{aligned}
$$

where (,) represents the time interval of the data. If a change-point $T_{0}$ is fixed based on external data or information, $\mathrm{AIC}_{0}$ should be compared with $\mathrm{AIC}_{12}=\mathrm{AIC}_{1}+$ $\mathrm{AIC}_{2}$. Otherwise, the arbitrary selection of the change point increases the degrees of freedom of the latter model and $\mathrm{AIC}_{0}$ must be compared with $\mathrm{AIC}_{12}=\mathrm{AIC}_{1}+\mathrm{AIC}_{2}+$ $2 k(n)$, where $k(n)$ is a penalty term estimated by a Monte Carlo simulation (Ogata, 1992).

When an earthquake catalog contains a swarm sequence, we divide it into three subsets: pre-swarm, swarm, and postswarm periods. In the present study, we consider the following four models. (1) Single ETAS model. This is a null hypothesis, one ETAS model with five parameters without change point. (2) Combined ETAS model. We divide the earthquake catalog into three subsets of different periods, pre-swarm, swarm, and post-swarm periods, and fit an ETAS model to each subset. This model has fifteen parameters with two change-points, $T_{\mathrm{cp} 1}$ and $T_{\mathrm{cp} 2}$, corresponding to the beginning and the end of the swarm period. We can classify each earthquake into two states which are swarm or not, however we can't determine where the real change point is between the last earthquake in the swarm period and 

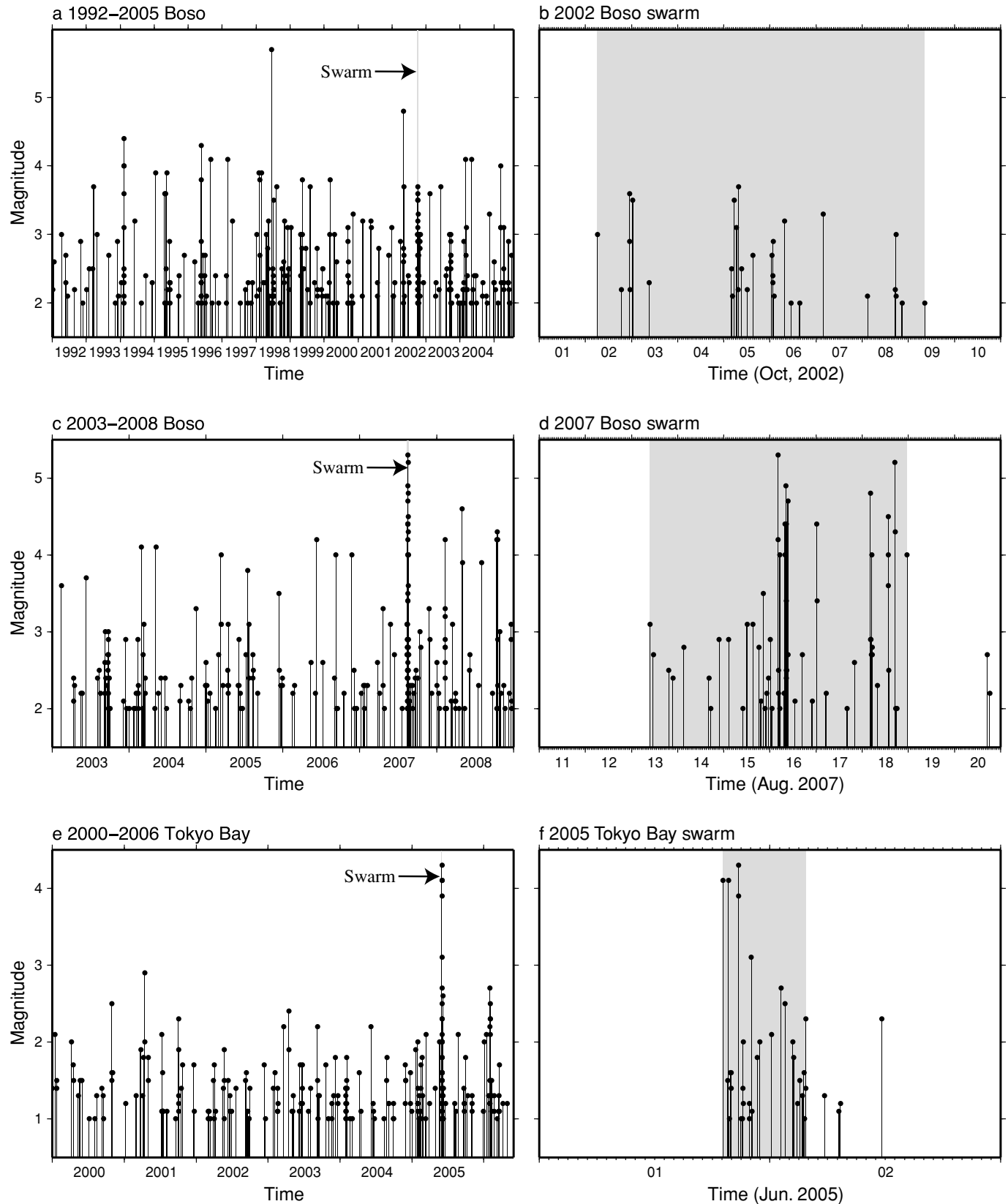

Fig. 2. The magnitude-time plot of seismicity. (a) Seismicity around the 2002 Boso swarm for 1992-2005. (b) The blow-up figure of (a). (c) Seismicity around the 2007 Boso swarm for 2003-2008. (d) The blow-up figure of (c). (e) Seismicity around the 2005 Tokyo Bay swarm for 2000-2006. In each panel, the swarm period is shown by gray area. (f) The blow-up figure of (e).

the first earthquake in the post-swarm period. Therefore we define $T_{\mathrm{cp} 2}$ as the hypocentral time of the last earthquake in the swarm period. (3) Boxcar swarm model. The background seismicity is $\mu_{0}$ except during the period of swarm, $T_{\mathrm{cp} 1}<t<T_{\mathrm{cp} 1}+T_{\mathrm{sw}}$, when the background rate is $\mu_{1}$. This hypothesis has seven parameters $\left(\alpha, c, K, p, \mu_{0}, \mu_{1}\right.$, and $T_{\mathrm{sw}}$ ) and one change-point $T_{\mathrm{cp} 1}$. Although we consider $T_{\mathrm{sw}}$ as one of the parameters, we may regard it as another change point, which we will discuss later. (4) Exponential swarm model. The background seismicity increases to $\mu_{1}$ at the beginning of the period of swarm $T_{\mathrm{cp} 1}$ and decreases exponentially as $\left(\mu_{1}-\mu_{0}\right) \exp \left(-t / T_{\mathrm{sw}}\right)+\mu_{0}$. The number of parameters are the same as the boxcar model. If swarm is a response to various external forces such as large static stress change due to magma intrusion, volcanic eruption, diffusive migration of fluid, and SSE, an exponential function may better represent transient change of seismicity.

\section{Data and Results}

\subsection{Earthquake catalogs}

We analyze swarm activity in two regions around the Boso Peninsula (Fig. 1). The one is the southeast of the peninsula, where slow slip events have been detected by GPS in 1996, 2002, and 2007. The swarm activities related with the last two SSEs were studied by Llenos et al. (2009). They divided the seismicity catalog into four periods, 2002 pre-swarm, 2002 swarm, 2007 pre-swarm, and 2007 swarm, and determined ETAS parameters for each period to study the temporal change of parameter values. We refer to these swarms as the Boso swarms. About 400 earthquakes equal 
Table 1. Earthquake catalogs. Time is shown by date (YYYY/MM/DD) and time (hh:mm). $N$ is the number of events and $M_{\mathrm{c}}$ is the minimum magnitude. Times shown by italic fonts are estimated values.

\begin{tabular}{cccccc}
\hline ID & $T_{\text {start }}$ & $T_{\text {end }}$ & $T_{\text {cp1 }}$ & $T_{\text {cp2 }}$ \\
\hline Boso02 & $1992 / 01 / 0722: 20$ & $2005 / 07 / 0904: 11$ & $2002 / 10 / 0206: 10$ & $2002 / 10 / 0908: 32$ & 300 \\
Boso07 & $2003 / 02 / 1301: 50$ & $2008 / 12 / 2020: 24$ & $2007 / 08 / 1309: 30$ & $2007 / 08 / 1823: 16$ & 250 \\
Tokyo05 & $2000 / 01 / 0115: 02$ & $2006 / 06 / 2501: 47$ & $2005 / 06 / 0119: 05$ & $2005 / 06 / 0203: 44$ & 250 \\
\hline
\end{tabular}

to or greater than $M_{\mathrm{J}}$ (local magnitude determined by Japan Meteorological Agency, JMA) 2.0 occurred from 1992 to 2007 , including two earthquakes greater than $M_{\mathrm{J}} 5.0$ during the 2007 SSE. The hypocenter locations during the two SSEs partially overlaps and the focal mechanisms determined by JMA are low angle thrust dipping to north or northwest. Many of these events have similar waveforms and identi ed as repeating earthquakes on the subducting plate interface by Kimura et al. (2006). Therefore, despite relatively large scatter in depth, $31.0 \mathrm{~km}$ in average with a standard deviation of $9.5 \mathrm{~km}$, in the original catalog, we consider that most of these earthquakes are interplate earthquakes.

Among many small clusters of earthquakes in Fig. 1, we focus on a swarm occurred beneath Tokyo Bay in 2005, which we refer to as the Tokyo Bay swarm. Figure 2 shows M-T plots for this cluster, together with those for two Boso swarms, as a long-period plot for years and its blow-up for the swarm period. The period of the Tokyo Bay swarm was short, from June 1st to 2nd, 2005, despite high seismicity, and there was no dominant mainshock. Four earthquakes greater than $M_{\mathrm{J}} 3.7$, among 38 detected earthquakes during these two days, and inactive aftershock seismicity following them imply that a simple ETAS model cannot explain this seismicity. While only six earthquakes greater than $M_{\mathrm{J}} 4.0$ occurred beneath Tokyo Bay from 2000 to 2009, it is curious that three of them are included in the swarm activity of this small cluster. This cluster has 250 events equal to or greater than $M_{\mathrm{J}} 1.0$ from January 2000 to June 2006. The focal mechanisms of the large four events are low-angle thrust dipping northward and consistent with the subduction direction of the Philippine Sea plate relative to the North American plate, $\mathrm{N} 30^{\circ} \mathrm{W}$. Hypocentral depths are distributed mainly between $25 \mathrm{~km}$ to $30 \mathrm{~km}$. These facts suggest that earthquakes in this cluster are interplate earthquakes similar to those in the Boso swarms (Matsubara et al., 2006).

We analyze these earthquake sequences by ETAS model and change-point hypothesis explained in the previous section. We neglect the spatial distribution and treat seismicity as a point process. Thus, a data set is a series of hypocentral time $t_{i}$ and magnitude $M_{i}$, from the JMA catalog, within a prescribed space and period. Each combined ETAS model requires two change points: the beginning and the end times of the swarm period, $T_{\mathrm{cp} 1}$ and $T_{\mathrm{cp} 2}$, respectively. The former is relatively easy to identify based on external information. Therefore, when the external information is available, we $\mathrm{x}$ the beginning time, while the latter is determined by minimizing the total AIC. The same beginning time is assumed for the change point $T_{\mathrm{cp} 1}$ for the boxcar swarm models.

For the Boso swarms, we prepare two time-magnitude catalogs that contain either the 2002 or the 2007 swarm event with an overlap period. For each period, the rst change point $T_{\mathrm{cp} 1}$ is xed to be the time of the rst event after the start time of the Boso SSE measured by GPS (Ozawa et al., 2003, 2007), while the second change point $T_{\mathrm{cp} 2}$ of the combined model is determined by MLE. For the Tokyo Bay swarm, we search both $T_{\mathrm{cp} 1}$ and $T_{\mathrm{cp} 2}$, because we have no external information. Each earthquake catalog is complete above a threshold magnitude $M_{\mathrm{c}}$, which means the magnitude-number statistics satis es the Gutenberg-Richter law (Gutenberg and Richter, 1954). Table 1 summarizes the beginning and the end of the catalog, $T_{\text {start }}$ and $T_{\text {end }}$, the xed change points common for all the models $T_{\mathrm{cp} 1}$, and the second change point for the combined model $T_{\mathrm{cp} 2}$, the threshold magnitude of the catalog $M_{\mathrm{c}}$, and the number of earthquakes $N$. It should be noted that the values shown by italic fonts in Table 1 are determined by MLE.

\subsection{The result of ETAS modeling}

Table 2 shows the ETAS parameters determined by MLE for three catalogs using combined ETAS models. For the swarm period, we adopted Omori law in place of OmoriUtsu law and xed the Omori's parameter $p$ to be one because this assumption gives smaller AIC. The con dence interval for each ETAS parameter is estimated as the interval that changes the maximum log-likelihood value by two units (see Appendix). Since we assume that every parameter has a log-normal distribution, the interval is given as the ratio as shown in Table 2 . Generally $K$ and $\mu$ are determined within a factor of 2, while $\alpha$ and $c$ have larger errors. Judging from the uncertainty, the difference of background seismicity $\mu$ is signi cant for three catalogs. In each Boso swarm, $\mu$ increases by two orders during the swarm, which is essentially the same result as Llenos et al. (2009), while the values are somewhat different, especially for $K$. The source of this difference is unclear, but one candidate is the insuf cient search for local minima due to the nonlinearity of MLE. The similar increase is observed for the Tokyo Bay swarm. The value of $\mu$ in the Tokyo Bay swarm is larger than that in the Boso swarms by one order.

Figure 3 shows the comparison between the cumulative number of events and the transformed time, which is the number of events by ETAS prediction, calculated using the pre-swarm parameters in Table 2. The increase of cumulative number after the change point $T_{\mathrm{cp} 1}$ is signi cant and cannot be explained by the pre-swarm ETAS parameters, but after the change point $T_{\mathrm{cp} 2}$, the slope is almost the same as the pre-swarm slope, as expected from the proximity between pre-swarm and post-swarm parameters. Thus the prediction from the combined models successfully explains the data, which suggests that we can identify the swarm period as a period of completely different characteristics with definite beginning and end times. 
Table 2. Calculated ETAS parameters by MLE. The number in round brackets is error ratio, assuming that each parameter has log normal distribution.

\begin{tabular}{|c|c|c|c|c|c|c|c|c|}
\hline & & $\alpha$ & $c$ & $K$ & $p$ & $\mu$ & $\mu_{1}$ & $T_{\mathrm{sw}}$ \\
\hline \multirow[t]{12}{*}{ Boso2002 } & Single & 0.53 & 0.0007 & 0.032 & 0.90 & 0.02 & - & - \\
\hline & & (1.28) & (4.19) & (1.16) & (1.04) & (1.37) & - & - \\
\hline & Pre & 0.67 & 0.0386 & 0.034 & 1.02 & 0.02 & - & - \\
\hline & & $(1.26)$ & $(2.50)$ & (1.24) & (1.09) & (1.30) & - & - \\
\hline & Swarm & $8 \times 10^{-10}$ & 0.0003 & 0.054 & $1.0^{*}$ & 2.21 & - & - \\
\hline & & $\left(8 \times 10^{8}\right)$ & (9.13) & (1.73) & - & (1.71) & - & - \\
\hline & Post & 0.17 & $2 \times 10^{-5}$ & 0.041 & 0.80 & 0.02 & - & - \\
\hline & & (3.39) & $(30.0)$ & $(1.31)$ & (1.09) & (1.93) & - & - \\
\hline & Boxcar & 0.60 & 0.0002 & 0.025 & 0.86 & 0.02 & 2.82 & 7.10 \\
\hline & & $(1.25)$ & $(2.00)$ & (1.17) & (1.04) & (1.37) & (1.59) & (1.38) \\
\hline & Exp. & 0.64 & 0.0002 & 0.023 & 0.87 & 0.02 & 3.93 & 6.32 \\
\hline & & (1.23) & $(2.00)$ & (1.17) & (1.05) & (1.34) & (1.54) & (1.43) \\
\hline \multirow[t]{12}{*}{ Boso2007 } & Single & 0.87 & 0.0019 & 0.019 & 1.09 & 0.05 & - & - \\
\hline & & (1.13) & (1.90) & (1.19) & (1.05) & (1.24) & - & - \\
\hline & Pre & 0.39 & 0.0001 & 0.019 & 0.93 & 0.05 & - & - \\
\hline & & $(2.08)$ & (7.48) & $(1.45)$ & (1.09) & $(1.28)$ & - & - \\
\hline & Swarm & 1.99 & 0.0011 & 0.002 & $1.0^{*}$ & 5.78 & - & - \\
\hline & & (1.07) & $(3.30)$ & (1.44) & - & $(1.45)$ & - & - \\
\hline & Post & 0.76 & 0.0019 & 0.024 & 1.04 & 0.05 & - & - \\
\hline & & (1.29) & (3.92) & (1.40) & (1.10) & (1.56) & - & - \\
\hline & Boxcar & 0.98 & 0.0009 & 0.011 & 1.09 & 0.05 & 5.86 & 5.35 \\
\hline & & (1.13) & $(2.11)$ & $(1.25)$ & $(1.05)$ & $(1.22)$ & (1.46) & (1.37) \\
\hline & Exp. & 0.62 & 0.0011 & 0.010 & 1.10 & 0.05 & 9.96 & 2.67 \\
\hline & & (1.12) & $(2.01)$ & (1.24) & (1.05) & $(1.22)$ & $(1.52)$ & (1.42) \\
\hline \multirow[t]{12}{*}{ Tokyo2005 } & Single & 1.03 & 0.0064 & 0.018 & 1.22 & 0.06 & - & - \\
\hline & & (1.12) & (1.66) & $(1.22)$ & (1.05) & $(1.20)$ & - & - \\
\hline & Pre & 0.18 & 0.0007 & 0.036 & 0.93 & 0.03 & - & - \\
\hline & & $(3.72)$ & $(4.61)$ & (1.28) & (1.08) & $(1.31)$ & - & - \\
\hline & Swarm & $2 \times 10^{-7}$ & 0.0001 & 0.032 & $1.0^{*}$ & 69.5 & - & - \\
\hline & & $\left(2 \times 10^{6}\right)$ & $(500)$ & $(2.48)$ & - & $(1.50)$ & - & - \\
\hline & Post & 1.00 & 0.0009 & 0.028 & 1.01 & 0.04 & - & - \\
\hline & & (1.39) & $(5.12)$ & $(1.40)$ & (1.10) & $(1.73)$ & - & - \\
\hline & Boxcar & 0.30 & 0.0008 & 0.030 & 1.00 & 0.04 & 70.00 & 0.36 \\
\hline & & (1.95) & (2.94) & $(1.24)$ & (1.06) & $(1.24)$ & (1.50) & (1.23) \\
\hline & Exp. & 0.22 & 0.0008 & 0.031 & 1.00 & 0.045 & 142.8 & 0.19 \\
\hline & & $(2.33)$ & $(2.91)$ & $(1.25)$ & (1.06) & $(1.23)$ & (1.49) & (1.37) \\
\hline
\end{tabular}

$(*)$ Fixed.

The change of background seismicity seems to be essential characteristics of a swarm sequence. If this is true, we may not have to change all 14 or 15 parameters of the combined model, because the best model maximizes the likelihood with small degrees of freedom. The simplest model would be the one only the background rate is variable, which is boxcar or exponential model. The parameters estimated for the boxcar and exponential models are also shown in Table 2. The end of the swarm period estimated for the boxcar model, $T_{\mathrm{cp} 1}+T_{\mathrm{sw}}$, does not have to match that of the combined model $T_{\mathrm{cp} 2}$, but the estimated end times are eventually identical. The transformed time calculated for the boxcar model is also almost proportional to the cumulative number (Fig. 3), and difference between combined and boxcar models is very small.

We calculate each AIC value considering the penalty of the change point problem (Table 3). The AIC for the single ETAS model (null hypothesis) is also calculated for the reference. Although all three models improve AIC values signi cantly from the null hypothesis, the boxcar model is
Table 3. Calculated AIC for each model. The AIC values include the change point penalties calculated by Monte Carlo simulation. The number in brackets is change point penalty term. The lower number of each cell is AIC improvement, which is the difference of AIC for combined ETAS and boxcar models from the single ETAS model.

\begin{tabular}{ccccc}
\hline & Single & Combined & Boxcar & Exponential \\
\hline Boso2002 & $1844.7(0)$ & $1807.3(9.3)$ & $1800.4(0)$ & $1799.7(0)$ \\
& 0 & -37.4 & -44.3 & -45.0 \\
Boso2007 & $881.4(0)$ & $822.8(9.1)$ & $814.1(0)$ & $823.0(0)$ \\
& 0 & -58.6 & -67.3 & -58.4 \\
Tokyo2005 & $1012.0(0)$ & $1005.1(24.7)$ & $981.4(9.1)$ & $984.2(9.1)$ \\
& 0 & -6.9 & -30.6 & -27.8
\end{tabular}

signi cant against the combined models in the Boso and Tokyo swarms. In this calculation the change point penalties are not added to the boxcar model. If we consider $T_{\mathrm{cp} 1}+T_{\mathrm{sw}}$ as a change point, the value of AIC increases by about 7, which is insuf cient to deny the superiority of the boxcar model. The exponential model has comparable 



Fig. 3. Comparison between the cumulative number of events and the transformed time, which is the predicted number of events by ETAS model, for (a) the 2002 Boso catalog, (b) the 2007 Boso catalog, and (c) the 2005 Tokyo Bay catalog. Red line shows the comparison using the pre-swarm parameters, extended to the whole catalog period. Green and purple lines show transformed time using swarm and post-swarm parameters, respectively. Blue line show transformed time using the boxcar model. Black and cyan dotted lines represent the beginning and the end times of swarm, $T_{\mathrm{cp} 1}$ and $T_{\mathrm{cp} 2}$, respectively. The black diagonal line is drawn as a reference. The corresponding M-T plots are shown in the lower panels.

values of AIC to those of the boxcar model for the 2002 Tokyo and the Boso swarms, suggesting the data are not sufficient enough to characterize the temporal change during the swarm period. However, the exponential model is not appropriate for the 2007 Boso swarm, for which the value of AIC is almost the same as that of the combined model. This is not surprising because many earthquakes occur in the latter half of the swarm period (Fig. 2(d)).

\section{Discussion and Conclusion}

The boxcar swarm model successfully explains seismicity rate change during a swarm. However the boxcar function is just one example and the other type of function may be better approximation. In fact, the exponential swarm model is comparable for the 2002 Boso and the 2005 Tokyo Bay swarms, but the specific shape of the function is not appropriate for the 2007 Boso swarm. Probably some swarm seismicity prefers a boxcar function with definite end time rather than an exponential function that decreases gradually. Although we have not tested other functions, we expect that it is difficult to find a simple function universally applicable for various swarm activity instead of a boxcar function. Therefore, we conclude that a boxcar function is preferable as the first degree assumption of swarm activity, and only the increase of the background seismicity is essential for swarm seismicity.

Since $\mu$ is related to the stressing rate (e.g., Llenos et al., 2009), we expect some external factors caused these swarms. The spatio-temporal correlation between the Boso swarms and SSEs strongly suggests that the SSEs triggered the swarms (NIED, 2007; Ozawa et al., 2007). Then what

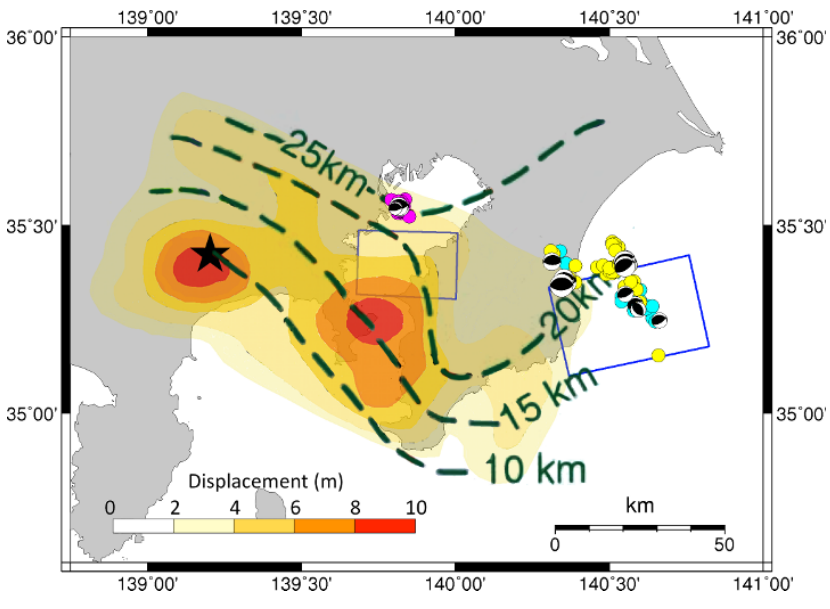

Fig. 4. Features around two swarm areas. Dashed lines represent the depth of the top of Philippine Sea plate and the contour from yellow to red means the slip of 1923 Kanto earthquake, which epicenter is black star (Sato et al., 2005). Blue rectangles adjacent to the Tokyo Bay and Boso swarm area show the 1989 Tokyo Bay SSE (Hirose et al., 2000) and the 2007 Boso SSE (NIED, 2007), respectively.

triggered the Tokyo Bay swarm? We propose the possibility of undetected SSE triggering the swarm from viewpoints of tectonic and seismic similarity. Both the Boso swarms and Tokyo swarm were located on the top of the Philippine Sea plate at depths of $20-30 \mathrm{~km}$, and the mechanisms of large earthquakes correspond with the subduction direction of the plate (Fig. 4). These locations were deep extent of the slip area of the 1923 Kanto Earthquake (Sato et al., 2005). The change of the background seismicity in these swarms is de- 
scribed by a boxcar function, although all swarm doesn't necessarily have these transient. For example, a swarm triggered by uid ow or magma intrusion might be described by a different function because these sources are more localized than SSEs.

It should be noted that this is not the rst paper that proposes the possibility of SSE beneath Tokyo Bay. Hirose et al. (2000) used tiltmeter and strainmeter records from 1985 to 1994 and a rectangular fault model to conclude that an SSE occurred beneath Tokyo Bay on December 9, 1989 at about $20 \mathrm{~km}$ south of the 2005 Tokyo Bay swarm area. The size of SSE is $M_{\mathrm{w}} 6.0$ and the duration is about one day. Although the seismic activity on the day is not high, we cannot derive de nite conclusion because the detection threshold at that time is worse than the current level. The relation between SSE and swarm may not be simple.

The stochastic analysis of seismicity little constrains the size of the possible SSE. However, if the SSE obeys the scaling law of slow earthquakes proposed by Ide et al. (2007), the size is determined simply by the duration. For the Boso swarms of about one week, the scaling law estimates the size of the corresponding SSE as $M_{\mathrm{w}}$ 5.8-6.5, which marginally covers the observational values of 6.46.6. The 1989 Tokyo Bay SSE determined by Hirose et al. (2000) is also about the upper limit of the predicted range. The duration of the 2005 Tokyo Bay swarm is about a half day, which corresponds to an SSE of $M_{\mathrm{w}}$ 5.0-5.7. This is close to the detection limit of SSE using Hi-net tiltmeters and very sensitive geodetic instruments can detect it. This size is small compared to the Boso events, but might have signi cant effects on stress accumulation to the source area of the next Kanto earthquake.

In summary, our study revealed that the swarm activities beneath the Boso peninsula and Tokyo Bay are successfully explained by a boxcar function swarm model including an ETAS model. Both swarms are located on the top of Philippine Sea plate and have mechanisms consistent with the plate motion. In addition to the well-observed SSEs corresponding to the Boso swarms, we expect a smaller undetected SSE for the Tokyo Bay swarm. This is small, but may be observable using today's best observational system.

Acknowledgments. We thank Y. Ogata for helpful discussions to understand history of point process model up to ETAS model and the AIC penalty of change point problem. We also thank the Japan Meteorological Agency for providing earthquake catalogs and the Generic Mapping Tools software freely distributed by Wessel and Smith (1991) for mapping the distribution of earthquakes and swarm sequences. Comments from two anonymous reviewers are helpful for revision. This work was supported by JSPS KAKENHI (20340115), MEXT KAKENHI (21107007), and Special Project for Earthquake Disaster Mitigation in Tokyo Metropolitan Area.

\section{Appendix A. The Confidence Interval of MLE}

In the point-process analysis, the Hessian matrix is written and approximated as follows:

$$
J_{i j}=\left.E_{X}\left[\frac{\partial^{2}}{\partial \theta_{i} \partial \theta_{j}} \log L(\theta)\right]_{\theta=\theta^{*}} \approx \frac{\partial^{2}}{\partial \theta_{i} \partial \theta_{j}} \log L(\theta)\right|_{\theta=\hat{\theta}}
$$

where $\theta^{*}$ is a set of genuine parameters and $\hat{\theta}$ is a set of MLE parameters. Then we calculate maximum log- likelihood around genuine parameters by using the Taylor series up to the second order,

$$
\begin{aligned}
\log L(\hat{\theta}) \approx & \log L\left(\theta^{*}\right)+\left.\Delta \theta_{i} \frac{\partial}{\partial \theta_{i}} \log L(\theta)\right|_{\theta=\theta^{*}} \\
& +\left.\frac{1}{2} \Delta \theta_{i} \Delta \theta_{j} \frac{\partial^{2}}{\partial \theta_{i} \partial \theta_{j}} \log L(\theta)\right|_{\theta=\theta^{*}} \\
\approx & \log L\left(\theta^{*}\right)+\frac{1}{2} \Delta \theta_{i} \Delta \theta_{j} J_{i j}
\end{aligned}
$$

where $\Delta \theta$ represents $\hat{\theta}-\theta^{*}$ and we use the Einstein notation and regard the rst order term is zero. In addition, the MLE parameters have asymptotically multivariate normal distribution as follows,

$$
\begin{aligned}
& \hat{\theta} \sim N\left(\theta^{*}, J^{-1}\right) \\
& f\left(\theta ; \theta^{*}, J^{-1}\right)=\frac{1}{(2 \pi)^{p / 2}\left|J^{-1}\right|^{1 / 2}} \exp \left[-\frac{1}{2} \Delta \theta_{i} \Delta \theta_{j} J_{i j}\right]
\end{aligned}
$$

We estimate the con dence interval of each ETAS parameter as the interval where the changes of the maximum log-likelihood value remains less than two. That is,

$$
\frac{1}{2} \Delta \theta_{i} \Delta \theta_{j} J_{i j}=2
$$

This criterion implies so-called 2-sigma rule.

\section{References}

Aoyama, H., M. Takeo, and S. Ide, Evolution mechanisms of an earthquake swarm under the Hida Mountains, central Japan, in 1998, J. Geophys. Res., 107, doi:10.1029/2001JB000540, 2002.

Fletcher, R., Practical Methods of Optimization, Vol. 1 and 2., Wiley, New York, 1980.

Gutenberg, B. and C. F. Richter, Seismicity of the Earth and Associated Phenomena, 2nd ed., 310 pp., Princeton Univ. Press, Princeton, N.J., 1954.

Hirose, I., I. Kawasaki, Y. Okada, T. Sagiya, and Y. Tamura, A silent earthquake of December 9, 1989, in the Tokyo Bay, as revealed by the continuous observation of crustal movements in the southern Kanto District, central Japan, Jishin, 53, 11-23, 2000.

Ide, S., G. C. Beroza, D. R. Shelly, and T. Uchide, A scaling law for slow earthquakes, Nature, 447, 76-79, 2007.

Kimura, H., K. Kasahara, T. Igarashi, and N. Hirata, Repeating earthquake activities associated with the Philippine Sea plate subduction in the Kanto district, central Japan: A new plate con guration revealed by interplate aseismic slips, Tectonophysics, 417, 101-118, 2006.

Llenos, A. L., J. J. McGuire, and Y. Ogata, Modeling seismic swarms triggered by aseismic transients, Earth Planet. Sci. Lett., 281, 59-69, 2009.

Lohman, R. B. and J. J. McGuire, Earthquake swarms driven by aseismic creep in the Salton Trough, California, J. Geophys. Res., 112, B04405, doi:10.1029/2006JB004596, 2007.

Matsubara, M., H. Kimura, K. Shiomi, and Y. Ito, Seismic activity beneath the Tokyo bay, Rep. Coord. Comm. Earthq. Predict., 75, 221-223, 2006.

Montgomery-Brown, E. K., P. Segall, and A. Miklius, Kilauea slow slip events: Identi cation, source inversions, and relation to seismicity, $J$. Geophys. Res., 114, B00A03, doi:10.1029/2008JB006074, 2008.

NIED, Seismic swarm activity off the east coast of the Boso peninsula, August 2007, Rep. Coord. Comm. Earthq. Predict., 79, 123-127, 2007.

Ogata, Y., Statistical models for earthquake occurrences and residual analysis for point processes, J. Am. Stat. Assoc., 83, 9-27, 1988.

Ogata, Y., Detection of precursory relative quiescence before great earthquakes through a statistical model, J. Geophys. Res., 97, 19845-19871, 1992.

Ogata, Y., Seismicity analysis through point-process modeling: A review, Pure Appl. Geophys., 155, 471-507, 1999.

Ohtake, M., Seismic activity induced by water injection at Matsushiro, Japan, J. Phys. Earth, 22, 163-176, 1974. 
Ozawa, S., S. Miyazaki, Y. Hatanaka, T. Imakiire, M. Kaidzu, and M. Murakami, Characteristic silent earthquakes in the eastern part of the Boso peninsula, Central Japan, Geophys. Res. Lett., 30, doi: 10.1029/2002GL016665, 2003.

Ozawa, S., H. Suito, and M. Tobita, Occurrence of quasi-periodic slow-slip off the east coast of the Boso peninsula, Central Japan, Earth Planets Space, 59, 1241-1245, 2007.

Reyners, M. and S. Bannister, Earthquakes triggered by slow slip at the plate interface in the Hikurangi subduction zone, New Zealand, Geophys. Res. Lett., 34, L14305, doi:10.1029/2007GL030511, 2007.

Sato, H., N. Hirata, K. Koketsu, D. Okaya, S. Abe, R. Kobayashi, M. Matsubara, T. Iwasaki, T. Ito, T. Ikawa, T. Kawanaka, K. Kasahara, and S. Harder, Earthquake source fault beneath Tokyo, Science, 309, doi:10.1126/science.1110489, 2005.

Segall, P., E. K. Desmarais, D. Shelly, A. Miklius, and P. Cervelli, Earthquakes triggered by silent slip events on Kilauea volcano, Hawaii, $\mathrm{Na}$ ture, 442, doi:10.1038/nature04938, 2006.
Toda, S., R. S. Stein, and T. Sagiya, Evidence from the AD 2000 Izu islands earthquake swarm that stressing rate governs seismicity, Nature, 419 , 58-61, 2002.

Utsu, T., A statistical study on the occurrence of aftershocks, Geophys. Mag., 30, 521-605, 1961.

Utsu, T., Y. Ogata, and R. S. Matsu'ura, The centenary of the Omori formula for a decay law of aftershock activity, J. Phys. Earth, 43, 133, 1995.

Vidale, J. E. and P. M. Shearer, A survey of 71 earthquake bursts across southern California: Exploring the role of pore uid pressure uctuations and aseismic slip as drivers, J. Geophys. Res., 111, B05312, doi:10.1029/2005JB004034, 2006.

Wessel, P. and W. H. F. Smith, Free software helps map and display data, Eos Trans. AGU, 72, 441, 1991.

T. Okutani and S. Ide (e-mail: ide@eps.s.u-tokyo.ac.jp) 